crescimento dos maxilares. Materiais e métodos: Foram utilizados registros das fichas clínicas de 300 bebés com até 6 meses de idade na primeira consulta e que foram acompanhados até no mínimo 12 meses de idade e no máximo 36 meses de idade, atendidos no Serviço de Atenção à Saúde Oral na Primeira Infância no município de Belém - Pará - Brasil; foi realizada análise estatística com o programa Statistical Package for the Social Sciences. Resultados: Resultados associados ao aleitamento materno e desenvolvimento do sistema estomatognático: largar o aleitamento materno mais tarde está associado a menores alteração de crescimento dos maxilares, alteração de posicionamento dental ( $\mathrm{p}<0,001$ em ambos os casos), alteração de mastigação ( $p=0,028)$; mas a associação não é significativa com a alteração na sequência de erupção $(p=0,172)$ e atraso na fala $(p=0,064)$. As alterações de crescimento dos maxilares e de posicionamento dental foram significativamente mais altas nas crianças que usaram algum tipo de sucção artificial ( $p<0,001$ em ambos os casos) Conclusões: O estudo conclui que largar o aleitamento materno mais tarde está associado a menores alteração de crescimento dos maxilares, alteração de posicionamento dental. A atenção à saúde oral na primeira infância, quando iniciada antes dos 6 meses de idade, pode contribuir para o retorno ao aleitamento materno exclusivo, promover o prolongamento do aleitamento materno, diminuir a prevalência da cárie, das alterações de crescimento dos maxilares e posicionamento dentário, bem como, possibilitar a identificação precoce das alterações do desenvolvimento infantil promovendo o acesso à assistência inclusiva.

http://doi.org/10.24873/j.rpemd.2019.12.550

\section{\#089 Descoloração dentária após endodontia regenerativa com cimentos de silicato de cálcio}

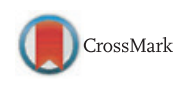

Joana Isabel de Pinho Santos*, Joana Alexandra Marques Simões, Rui Isidro Falacho, Francisco Caramelo, João Miguel dos Santos, Paulo Jorge Rocha da Palma

Instituto de Endodontia da Faculdade de Medicina da Universidade de Coimbra, Área de Medicina Dentária Faculdade de Medicina da Universidade de Coimbra, Instituto de Implantologia e Prostodontia da Faculdade de Medicina da Universidade de Coimbra, Laboratório de Bioestatística e Informática Médica da Faculdade de Medicina Universidade de Coimbra

Objetivos: Avaliar e comparar a descoloração coronal induzida por três cimentos de silicato de cálcio utilizados em procedimentos regenerativos - TotalFill BC RRM putty (FKG), Biodentine (Septodont) and ProRoot MTA (Dentsply). Materiais e métodos: Quarenta e dois dentes foram seccionados perpendicularmente ao seu longo eixo (2 $\mathrm{mm}$ aquém da junção amelocementária) e preparados a partir da sua porção apical. Após preparação do acesso à câmara pulpar, as amostras foram divididas em 8 grupos: grupo 1, controlo negativo $(n=3)$; grupo 2, controlo positivo $(n=3)$; grupo 3, TotalFill BC RRM putty soro $(\mathrm{n}=6)$; grupo 4, TotalFill BC RRM putty sangue $(n=6)$; grupo 5 , Biodentine soro $(n=6)$; grupo 6, Biodentine sangue $(n=6)$; grupo 7, ProRoot MTA soro $(n=6)$; grupo 8, ProRoot MTA sangue $(n=6)$. Após preenchimento com o biomaterial, os dentes foram res- taurados com SDR Flow Bulk Fill Flowable A2 (Dentsply Caulk, Milford, USA). A cor foi avaliada no momento inicial (previamente à colocação do biocerâmico - T0), bem como imediatamente (TPO), 72 horas (T72H), 7 dias (T7D) e 1 mês (T1M) após aplicação do biomaterial, utilizando o Sistema $\mathrm{L}^{*} \mathrm{a}^{*} \mathrm{~b}^{*}$ da Comission International de l'Eclairage. A variação de cor, $\Delta \mathrm{E}$, foi comparada entre os diferentes grupos e ao longo do tempo, com nível de significância $p<0,05$. Resultados: Verificaram-se diferenças estatisticamente significativas relativamente à variação de cor dos biomateriais ao longo do tempo $(\mathrm{p}<0,001)$, tendo em conta o material, o tratamento (sangue ou soro) ou ambos. Se apenas o material ou o tratamento forem considerados, independentemente do tempo, não são encontradas diferenças estatisticamente significativas $(p=0,111)$. Relativamente ao tempo de avaliação T1M, existem diferenças significativas entre os grupos ( $p<0,001)$, sendo que, o Biodentine é o material que exibe melhor estabilidade de cor seguido pelo TotalFill BC e MTA. O MTA apresenta a maior variação de cor, tendo significância estatística para os restantes materiais $(p<0,001)$. Conclusões: Após um período de avaliação de 1 mês, a contaminação do material por sangue pode não ser um fator crítico para a descoloração dos biomateriais. O Biodentine é o cimento de silicato de cálcio que apresenta maior estabilidade de cor e o MTA o que apresenta menor, conduzindo por esse motivo a uma maior descoloração dentária ao longo do tempo. Assim, a seleção do biomaterial deve ter em consideração o potencial de descoloração inerente. http://doi.org/10.24873/j.rpemd.2019.12.551

\#090 Maturação da Sutura Palatina Mediana: estudo comparativo entre dois métodos de avaliação

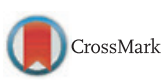

Mariana da Rocha e Almeida*, Eugénio Martins, Sofia Isabel Madeira Reis, Ana Catarina Remoaldo Vaz, Saul Castro, Maria João Ponces

Faculdade de Medicina Dentária da Universidade do Porto

Objetivos: Este estudo retrospetivo visou validar a utilização do método BOKA, um método proposto recentemente para a avaliação da maturação da sutura palatina mediana através de radiografias panorâmicas, pela sua comparação com um método de avaliação da sutura em cortes de tomografia computorizada de feixe cónico, proposto por Angelieri et. al. (Angelieri, 2013). Colocou-se como hipótese nula a não existência de correlação entre os métodos de BOKA e de Angelieri et. al. Materiais e métodos: A amostra, constituída por 76 indivíduos (32 do sexo masculino e 44 do sexo feminino, com uma média de idade de 18,8 \pm 11,6 anos), foi selecionada aleatoriamente a partir dos registo clínicos de primeiras consultas consecutivas de ortodontia que preenchiam os critérios de inclusão e que realizaram tomografia computorizada de feixe cónico como meio complementar de diagnóstico numa clínica privada de ortodontia. Foram produzidas 152 secções de tomografia computorizada de feixe cónico, sendo que 76 correspondiam a cortes axiais, onde se avaliou a maturação da sutura pelo método de Angelieri et. al, e 76 correspondiam a projeções do tipo panorâmica, onde se avaliou a maturação da sutura pelo método de BOKA. Os scans foram analisados pelos 2 exami- 
nadores, que foram devidamente treinados e realizaram um exercício de calibração antes da análise. Passadas 2 semanas foi feita uma nova análise em 30 scans escolhidos aleatoriamente, de modo a permitir a análise do erro intra-examinador. O Coeficiente de Kappa foi utilizado para avaliar o erro intra e inter examinador. O Coeficiente de Correlação de Spearman e - Coeficiente de Correlação Intraclasses foram utilizados para avaliar a correlação entre as 2 escalas. O tratamento estatístico dos dados foi realizado com recurso ao software SPSS ${ }^{\circledR}$. Considerou-se um nível de significância de 5\%. Resultados: Os valores do Coeficiente de Kappa foram maiores que 0,85, tanto na avaliação do erro inter como do erro intra observador, para ambas as escalas. Os valores do Coeficiente de Correlação de Spearman $(0,838)$ e do Coeficiente de Correlação Intraclasses $(0,883)$ mostram a existência de uma forte correlação entre as 2 escalas. Conclusões: Os resultados deste estudo demonstram que as escalas de BOKA e Angelieri et al. são ambas reprodutíveis e que têm uma forte correlação entre si, o que leva à conclusão de que a escala BOKA parece ser efetiva na avaliação do grau de maturação da sutura palatina.

http://doi.org/10.24873/j.rpemd.2019.12.552

\section{\#091 Avaliação da largura cortical mandibular em TCs de pessoas com doença falciforme}

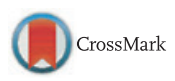

Renata da Paz Leal Pereira*, Juliana Jorge Garcia, Emi Suzane de Abreu Dias, Viviane de Almeida Sarmento, Heloísa Laís Rosario dos Santos, Viviane de Sousa Moreira Almeida

Universidade Federal da Bahia

Objetivos: O objetivo do presente estudo foi avaliar a largura da cortical mandibular (LCM) em indivíduos portadores de doença falciforme. Materiais e métodos: Setenta e cinco reconstruções panorâmicas de tomografias computadorizadas de pacientes portadores de doença falciforme foram coletadas para a análise da Largura da Cortical Mandibular na região mentual (LCM). A análise foi realizada por dois avaliadores. Em cada exame, o LCM foi medido dos lados da mandíbula, duas vezes por um mesmo avaliador, com intervalo de no mínimo sete dias entre as avaliações. Para avaliação do LCM, foi medido o valor de ' $C$ ' que corresponde a altura da cortical mandibular abaixo do forame mentual. Os dados foram registrados em uma tabela e enviados para posterior análise estatística. Resultados: Inicialmente foi calculada a média das duas medidas realizadas em cada lado da mandíbula pelo avaliador e, em seguida, foi calculada a média do LCM entre os dois lados da mandíbula de cada paciente. O valor de LCM foi considerado normal quando igual ou maior a $4 \mathrm{~mm}$. Os resultados revelaram uma média de LCM de $4,1 \mathrm{~mm}$ (com desvio-padrão de $0,7 \mathrm{~mm}$ ). Vinte e sete pacientes $(36,5 \%)$ apresentaram valores de LCM diminuídos e 47 tiveram valores considerados normais (63,5\% da amostra) Discussão: Os resultados demonstraram que na amostra avaliada uma grande parte dos pacientes já apresentavam diminuição da massa óssea mandibular detectada por este índice. Radiografias odontológicas podem revelar o efeito que a doença falciforme tem sobre o metabolismo ósseo, evidenciando perdas ósseas e más oclusões decorrentes da hiperplasia medular compensatória. Conclusões: A largura da cortical mandibular pode ser utilizada para avaliar osteopenia resultante de diversas condições sistêmicas e nos Indivíduos com doença falciforme este índice pode demonstrar a presença de alterações ósseas. http://doi.org/10.24873/j.rpemd.2019.12.553

\section{\#92 Influência da anestesia: Subperióstea} Vs Loco-regional - Ensaio Clínico Aleatorizado

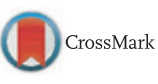

Ana Rita Jorge Reis*, André Chen, Helena Francisco, Joana Fialho, João Caramês

Faculdade de Medicina Dentária da Universidade de Lisboa, Escola Superior de Tecnologia e Gestão de Viseu; Centro de Estudos em Educação, Tecnologia e Saúde

Objetivos: Quando é necessário realizar exodontia de 1os ou 2os molares mandibulares a escolha da técnica anestésica da maioria dos profissionais de saúde oral irá recair sobre o bloqueio loco-regional, no entanto, com o aparecimento da articaína pode ser possível alcançar uma anestesia eficaz pela técnica subperióstea. O objetivo deste estudo é comparar a eficácia anestésica do Cloridrato de articaína a 4\% com epinefrina a 1:200 000 na exodontia de 1os e 2os molares mandibulares quando utilizada anestesia subperióstea vs. anestesia loco-regional. Materiais e métodos: Ensaio clínico aleatorizado com 15 pacientes, que se dirigiram à clínica universitária da Faculdade de Medicina Dentária da Universidade de Lisboa, para a extração de 1 os ou 20 molares mandibulares. Após aleatorização os pacientes receberam anestesia loco-regional ou anestesia infiltrativa de acordo com o grupo atribuído. De forma a determinar a eficácia anestésica alcançada para cada uma das técnicas, foi registada numa escala VAS (Visual Analogue Scale) a dor sentida antes, durante e após o procedimento e a dor durante a anestesia. Foi registada pelo clínico a quantidade de anestésico utilizado. Resultados: Não foram encontradas diferenças estatisticamente significativas $(p>0,05)$ quando se compara a dor sentida antes, durante e após o procedimento e a dor durante a anestesia. Foram verificadas diferenças estatisticamente significativas $(p<0,05)$ entre a quantidade de anestésico utilizado pelas duas técnicas. Conclusões: Os resultados deste estudo sugerem que não existe diferença na eficácia anestésica do Cloridrato de articaína a 4\% com epinefrina a 1:200 000 na exodontia de 1 os e 20 s molares mandibulares quando utilizada anestesia subperióstea vs. anestesia loco-regional. http://doi.org/10.24873/j.rpemd.2019.12.554

\section{\#093 Endocrown Vs. Restauração parcial cerâmica com pino de fibra - Estudo clínico randomizado \\ CrossMark}

Susana Morimoto*, Reinaldo Macedo Fraga, Tamara Kerber Tedesco, Mutlu Özcan, Maria Stella Nunes A Moreira, Daniela Prócida Raggio

Universidade de São Paulo- FOUSP, University of Zürich, Switzerland, UniversidadeIbirapuera, São Paulo, Brasil, Universidade Ibirapuera, São Paulo, Brasil

Objetivos: Comparar o tempo operatório, desconforto e satisfação de pacientes restaurados com endocrowns cerâmi- 